\title{
Pulsed x-ray imaging of high-density objects using a ten picosecond high- intensity laser driver
}

D. Rusby, C. Brenner, C. Armstrong, L. Wilson, R. Clarke, et al.

D. R. Rusby, C. M. Brenner, C. Armstrong, L. A. Wilson, R. Clarke, A. Alejo, H. Ahmed, N. M. H. Butler, D. Haddock, A. Higginson, A. McClymont, S. R. Mirfayzi, C. Murphy, M. Notley, P. Oliver, R. Allott, C. Hernandez-Gomez, S. Kar, P. McKenna, D. Neely, "Pulsed x-ray imaging of high-density objects using a ten picosecond high-intensity laser driver," Proc. SPIE 9992, Emerging Imaging and Sensing Technologies, 99920E (25 October 2016); doi: $10.1117 / 12.2241776$

SPIE. Event: SPIE Security + Defence, 2016, Edinburgh, United Kingdom 


\title{
Pulsed X-ray imaging of high-density objects using a ten picosecond high- intensity laser driver
}

\author{
D. R. Rusby ${ }^{1,2}$, C. M. Brenner ${ }^{1}$, C. Armstrong ${ }^{1,2}$, L. A. Wilson ${ }^{1}$, R. Deas ${ }^{5}$, D. Lockley ${ }^{5}$, S. \\ Dorkings $^{5}$, K. Butler ${ }^{5}$, G. Cook ${ }^{5}$, M. Carpenter ${ }^{5}$, R.Giordmaina ${ }^{5}$, R. Clarke ${ }^{1}$, A. Alejo ${ }^{3}$, H. Ahmed , $^{3}$ \\ N. M. H. Butler ${ }^{2}$, D. Haddock ${ }^{1}$, A. Higginson ${ }^{2}$, A. McClymont ${ }^{1}$, S. R. Mirfayzi ${ }^{3}$, C. Murphy ${ }^{4}$, M.

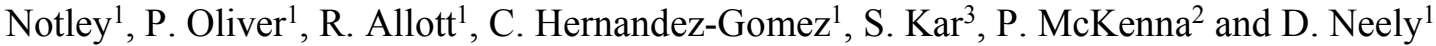 \\ ${ }^{1}$ Central Laser Facility, STFC, Rutherford Appleton Laboratory, Didcot, Oxon, OX11 0QX, UK \\ ${ }^{2}$ Department of Physics, SUPA, University of Strathclyde, Glasgow G4 0NG, UK \\ ${ }^{3}$ Centre for Plasma Physics, Queen's University Belfast, Belfast BT7 1NN, UK \\ ${ }^{4}$ Department of Physics, University of York, York YO10 5DD, UK \\ ${ }^{5}$ Security Sciences Department, DSTL, Fort Halstead, Sevenoaks, Kent, UK
}

\begin{abstract}
Point-like sources of X-rays that are pulsed (sub nanosecond), high energy (up to several MeV) and bright are very promising for industrial and security applications where imaging through large and dense objects is required. Highly penetrating X-rays can be produced by electrons that have been accelerated by a high intensity laser pulse incident onto a thin solid target. We have used a pulse length of $\sim 10 \mathrm{ps}$ to accelerate electrons to create a bright $\mathrm{x}$-ray source. The bremsstrahlung temperature was measured for a laser intensity from $8.5-12 \times 10^{18} \mathrm{~W} / \mathrm{cm}^{2}$. These $\mathrm{x}$-rays have sequentially been used to image high density materials using image plate and a pixelated scintillator system.
\end{abstract}

\section{INTRODUCTION}

High power laser-solid interactions have been shown to be a bright source of high energy ions ${ }^{1,2}$, neutrons ${ }^{3,4}$ and $\mathrm{x}$ rays $^{5,6,7}$. In the regime where the intensity of the laser is greater than $10^{18} \mathrm{~W} / \mathrm{cm}^{2}$, mega-Ampere Maxwellian distributed electron currents are accelerated and driven into the target. This electron current can be used to create a source of high energy Bremsstrahlung emission with temperatures up to several $\mathrm{MeV}^{7,8,9,10}$. The use of non-destructive testing of material through x-ray radiography is highly applicable to many industrial sectors. It is common for laser-solid interactions to be performed at the maximum irradiance of the laser which, for the laser system described herein, is typically using a pulse duration of $1 \mathrm{ps}$. The pulse duration of the subsequent x-ray emission, as a result is short pulsed. The emission area is also small $\left(<<1 \mathrm{~mm}^{2}\right)$ leading to radiography images with high resolution. All these qualities lead to a source of $\mathrm{x}$-rays that is ideal to perform high resolution projection $\mathrm{x}$-ray radiography of dense samples ${ }^{4,11}$. These qualities on a single shot cannot be matched by commercially available conventional sources of x-rays.

To optimize the creation of bremsstrahlung x-rays from electrons travelling through solid targets, a number of considerations must be made in regards the relationship between the target material, density and the source of electrons as these all directly affect the bremsstrahlung from the target. In the case of electrons in a laser solid interaction, the majority of electrons are confined to the target due to extremely high electric fields set up at the rear of the thin target $(\leq 1$ $\mathrm{mm})$. This results in the majority of the electrons $(\sim 95 \%)$ being reflected and reinjected once they reach the rear of the target ${ }^{12}$; which is known as refluxing. These electrons can reflux many times, depending on their energy and the material/thickness of the target, until they lose all their energy. As the electrons reflux they also diverge and continue to produce bremsstrahlung; which will increase the size of the source, deteriorating the resolution of the radiography. To reduce this effect, a target that is relativity thick with a high density can be used as well as reducing the energy/temperature of the electrons to increase the likelihood of them stopping on the first pass (ie. before they reflux). The temperature of these electrons, and therefore the x-rays, has been shown to depend on the intensity of the laser ${ }^{13,14,15}$; which depends on the energy, pulse length and the focal spot size. Reducing the focal spot area is ideal for small sources, whilst also keeping the energy high optimizes the total flux. The only parameter that will not be directly detrimental to the total flux and resolution of the x-rays is the pulse length. It has also been shown that the angular divergence of the

\footnotetext{
Emerging Imaging and Sensing Technologies, edited by Keith L. Lewis,

Richard C. Hollins, Proc. of SPIE Vol. 9992, 99920E · (C) 2016 SPIE

CCC code: $0277-786 \mathrm{X} / 16 / \$ 18 \cdot$ doi: $10.1117 / 12.2241776$
} 
electrons inside the material is proportional to the intensity, therefore reducing the laser intensity by increasing the pulse length will reduce the divergence ${ }^{16}$.

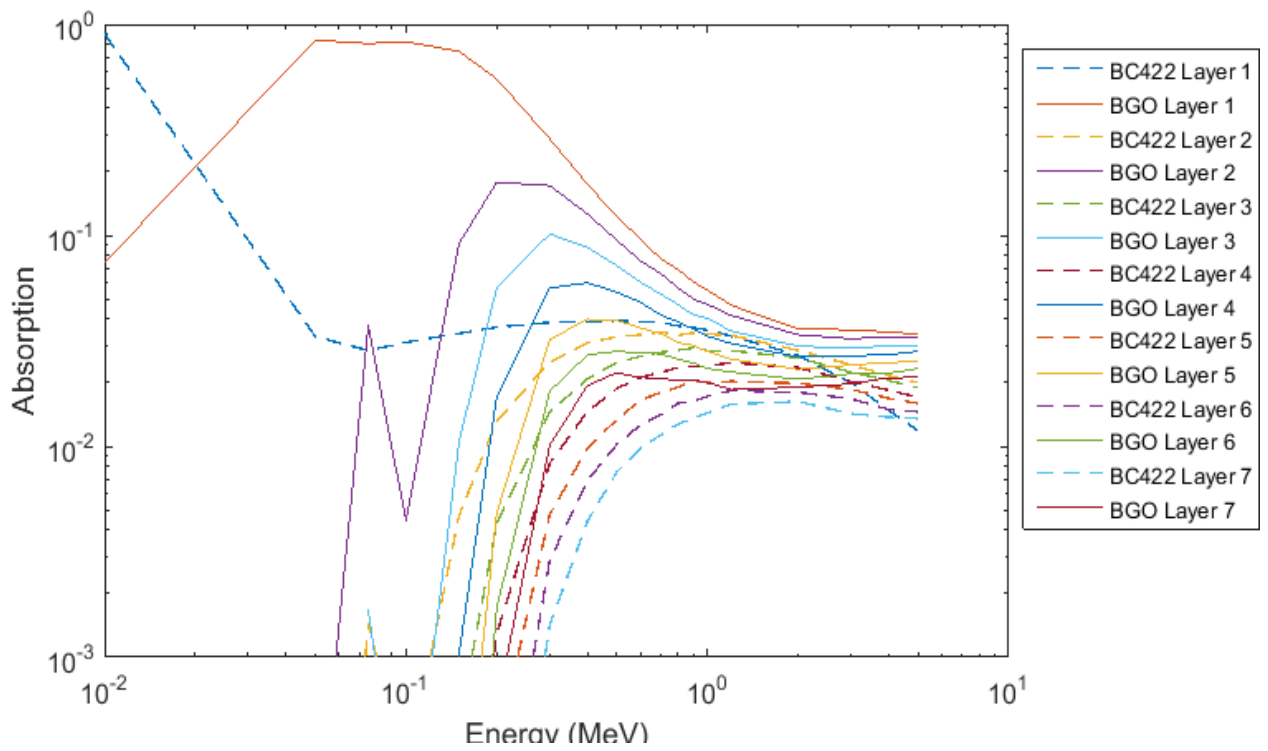

Figure 1: The $\mathrm{x}$-ray response curve of an array of $2 \mathrm{~mm} \mathrm{BGO}$ and $12 \mathrm{~mm} \mathrm{BC} 422$ produced using the Monte Carlo code Geant 4 . Each simulation was carried out with $10^{5}$ incident mono-energetic x-ray.

Many techniques are available to diagnose the properties of these x-rays. The angular distribution of the x-rays will be similar to that of the electrons emitted from the rear of the target, which can be measured using a diagnostic that consists of imaging plate that wraps around the rear of the target ${ }^{17,18}$. The spectral distribution of the x-rays, which is dictated by the temperature of the fast electrons and the transmission of the target, can be found using diffraction-crystal spectrometers $^{19}$ or nuclear activation ${ }^{20,21}$. However, diffraction-crystal spectrometers have a limited spectral range (upto $100 \mathrm{keV})$ and activation only samples the highest energies $(>10 \mathrm{MeV})$ and often requires long processing times to read the radioactive decay. The most widely used method of spectral analysis for laser-solid interactions is to use an absorption based spectrometer ${ }^{22,23}$. These work by placing sensitive material between filtering in a 1D array. These spectrometers normally use Imaging Plate (IP) which requires processing after every shot to determine the spectral information. A scintillator based version of this spectrometer has been developed to provide fast feedback of the spectral information on a shot to shot basis ${ }^{24}$. In this paper I will discuss how the spectrometer works and present the results obtained using a laser system with a pulse length of $10 \mathrm{ps}$. Finally a small portfolio of recorded images will be shown that demonstrate the imaging capabilities of such a source.

\section{SCINTILLATOR BASED SPECTROMETER}

As mentioned before, the most common technique for observing x-ray temperatures above $100 \mathrm{keV}$ is to use an absorption spectrometer. These spectrometers often use Imaging plate and high density filters to separate the spectral bins that the spectrometer can detect. This use of high density material reduces the efficiency of the detector; since signal is lost in the filtering layers. Using a scintillator to both act as the detector of the x-rays and also the attenuator increases the sensitivity. The scintillators are placed in a 1D array and imaged using a high sensitivity camera. The flexibility of scintillator thickness and density allow for a number of different spectrometers to be designed for optimum performance in characterizing different sources. 
The scintillators used in this experiment were BGO and BC422. BGO has a high density and high $\mathrm{Z}$ whereas $\mathrm{BC} 422$ has low density and low $\mathrm{Z}$. The variations in material type allows for different spectral sensitivity as the attenuation curves for each material are vastly different.

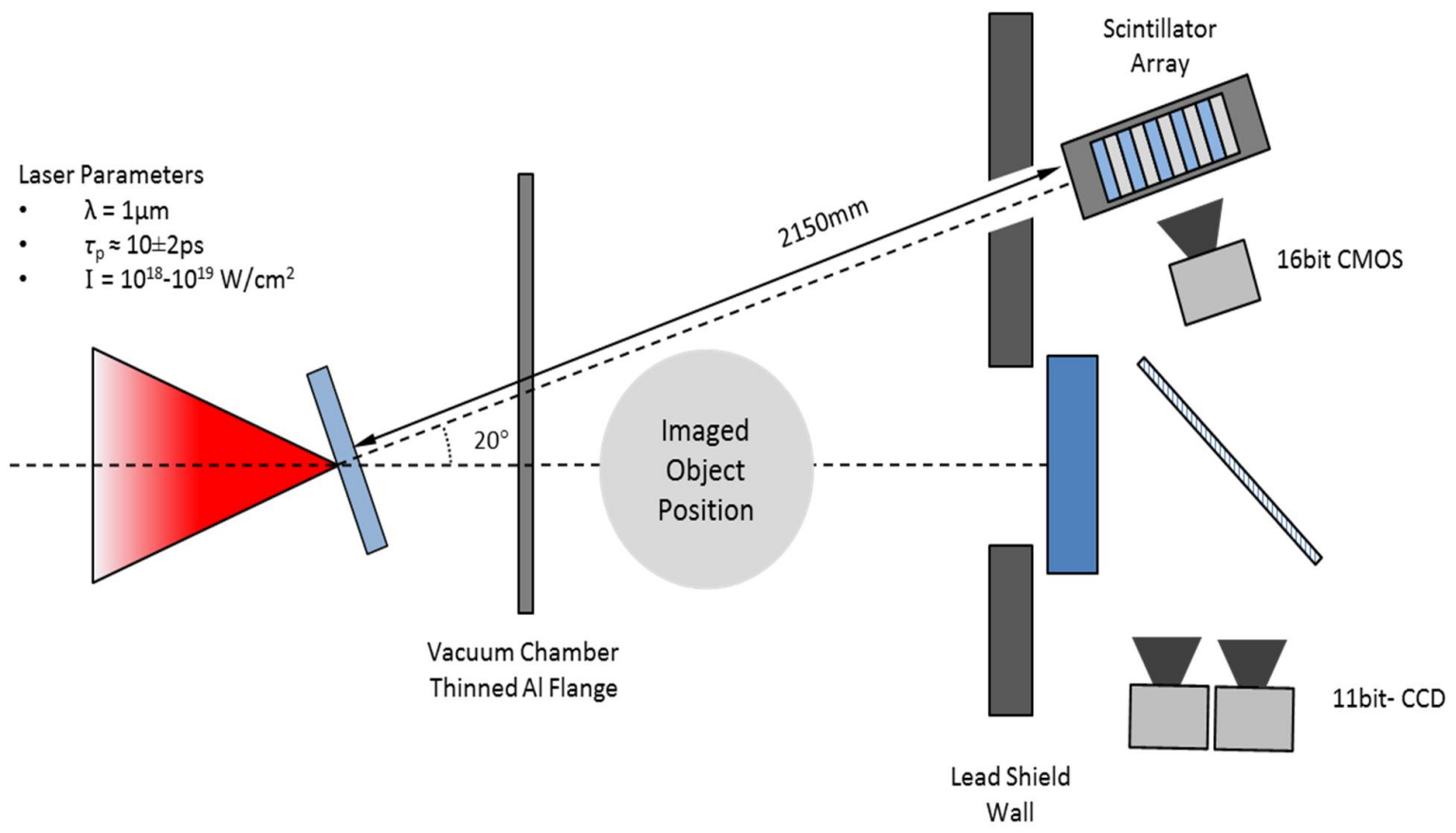

Figure 2: Schematic of the experimental setup used to measure the temperature of the x-rays and also use them to radiograph objects.

The Monte Carlo code GEANT4 ${ }^{25}$ was used to calculate the response of the diagnostic to x-rays. An array of alternating BGO and BC422 scintillators were built in GEANT4 with thicknesses of $2 \mathrm{~mm}$ and $12 \mathrm{~mm}$ respectively. The height and width was $30 \times 12 \mathrm{~mm}$ for both. To calculate the response curve mono-energetic x-rays were sent into the array. Multiple runs were conducted with different input energies. The final response curves are shown in Figure (1).

\section{EXPERIMENTAL METHOD/RESULTS}

Radiography and spectral measurements were made on an experiment using the Vulcan Laser ${ }^{26}$ at the Central Laser Facility (CLF) based at the Rutherford Appleton Laboratory. A schematic of the experimental setup is shown in Figure

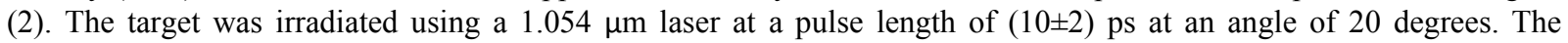
maximum laser energy incident onto target was $(140 \pm 15) \mathrm{J}$. The focal spot size was $\sim 7 \mu \mathrm{m}$ FWHM in diameter. The peak intensity achievable was $\sim 1.2 \times 10^{19} \mathrm{~W} / \mathrm{cm}^{2}$. The scintillator array was placed along the target normal direction, and just over $2 \mathrm{~m}$ from the interaction behind a lead shielding wall. The scintillator array is imaged using a 16-bit CMOS camera. The imaged objects were placed much closer to the interaction, just outside the vacuum chamber, to allow for projection imaging with high magnification.

Firstly a laser energy $\left(\mathrm{E}_{\mathrm{L}}\right)$ scan was performed on $1 \mathrm{~mm} \mathrm{Al}$ with the flux results shown in Figure (3) a). The flux recorded on the first layer of BGO scales as $\mathrm{E}_{\mathrm{L}}^{(1.8 \pm 0.2)}$ indicating that it is very sensitive to incident laser energy which was therefore kept as high as possible when performing radiography to create the maximum flux of x-rays. To extract the temperature from the data, a number of Geant4 simulations were initially conducted to find the x-ray spectrum from different input electron distributions 

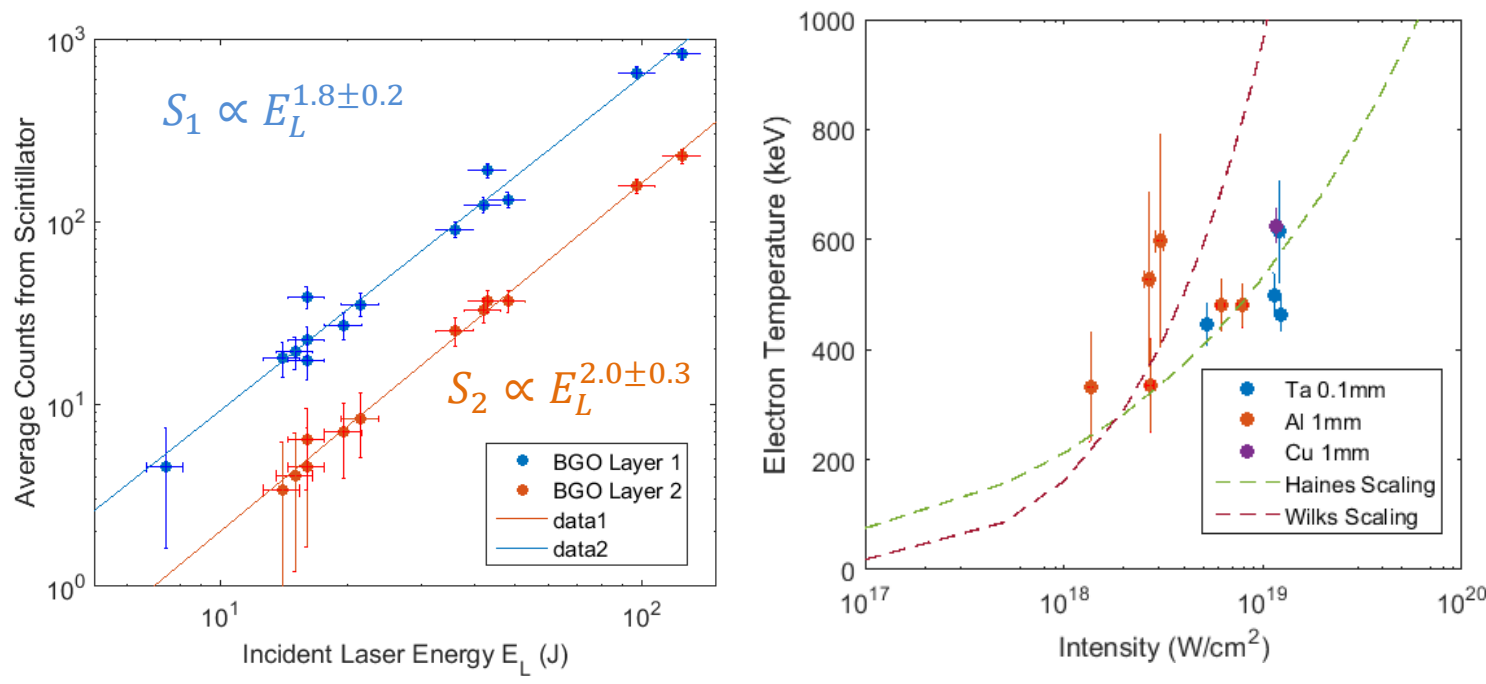

Figure 3: The average counts on the layers of scintillator as a function of incident laser energy $(\mathrm{J}), \mathrm{E}_{\mathrm{L}}$, onto the target. For both layers a fit power laws with slight different gradients is shown. b) shows the extracted temperature for 3 different targets as a function of incident laser intensity. Also plotted is the Haines and Wilks scaling.

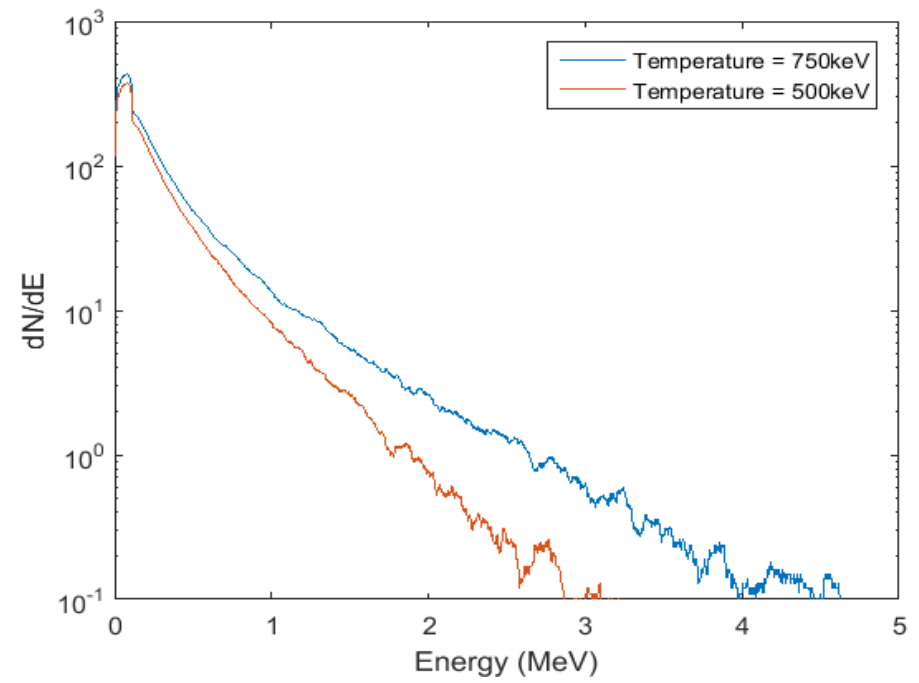

Figure 4: Output x-rays from Geant4 for $500 \mathrm{keV}$ and $750 \mathrm{keV}$ electron temperatures incident onto a $0.1 \mathrm{~mm}$ Ta target.

To calculate the temperature from the scintillator array, the simulated data is compared to the experimental data using the least squared fit. The source of the uncertainties in the measurement arises from the error in the background, which is taken as the standard deviation over many images, and the single pixel error from the camera. The extracted temperatures are shown in Figure (3) b) as a function of incident laser intensity. Also plotted in Figure(3) b) is the Haines and Wilks scaling laws that estimate the temperature as a function of intensity ${ }^{14,15}$. The experimental data is of a similar temperature to the scaling so shows a general increasing trend with intensity. The highest intensity experimental data has an electron temperature in the region of $500 \mathrm{keV}$ to $750 \mathrm{keV}$; these spectra are shown for a $0.1 \mathrm{~mm}$ Ta target in Figure (4) as this target was primarily used for imaging in later sections. 

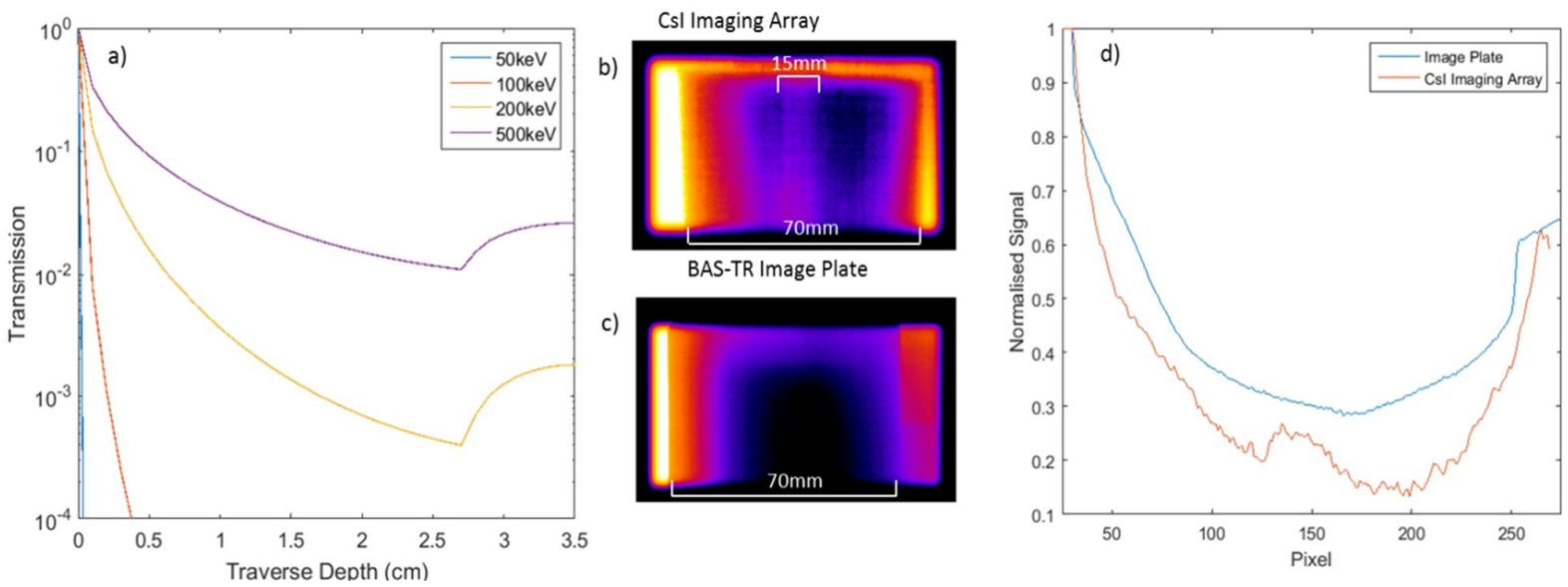

Figure 5: a) The transmission of a steal cylinder with an outer diameter of $7 \mathrm{~cm}$ and inner diameter of $1.5 \mathrm{~cm}$ for different input $\mathrm{x}$-ray energies. b) and c) show the images of the object described taken on the CsI imaging array and image plate respectively. It is clear that the inner hole is only visible using the CsI array. Taking a lineout of the images clearly shows the visibility of this feature, as shown in d).

\section{X-RAY RADIOGRAPHY}

$\mathrm{X}$-ray radiography of numerous objects was carried out using the source of $\mathrm{x}$-rays described above. The majority of images were recorded using a $0.1 \mathrm{~mm}$ Ta target. The images were recorded using two types of detector. BAS-SR image plate is a material with an active layer where electrons can be excited into a metastable state. This layer is thin so although it is has highly sensitive, it is mainly sensitive to low energy $\mathrm{x}$-rays. The second diagnostic was a $30 \times 30 \mathrm{~cm}$ Cesium Iodide Thallium-doped (CsI(Tl)) scintillator with $0.5 \times 0.5 \mathrm{~mm}$ pixel width with a depth of $1 \mathrm{~cm}$. To optimise the amount of light captured from the CsI array, two large-area 11bit-CCDs were used. One of major advantage of the CsI array is that it instantly returns an image whereas the image plate requires scanning after every shot. The thickness of the CsI provides much larger attenuation and therefore it can detect higher energy x-rays.

To demonstrate the energy sampling of each detector a steel cylinder with an outer diameter of $7 \mathrm{~cm}$ and inner hollow region of diameter $1.5 \mathrm{~cm}$ was imaged using both image plate and the CsI imaging array simultaneously. Figure (5) a) shows the attenuation of the material as a function of depth; the lower energy x-rays are instantly attenuated and the feature is only visible with $\mathrm{x}$-rays with energies greater than $200 \mathrm{keV}$. As mentioned before the image plate is primarily sensitive to less than $100 \mathrm{keV}$ with a peak sensitivity at $\sim 50 \mathrm{keV} \mathrm{x}$-rays which do not transmit, which is clear from the picture as the inner hollow region is not visible on Figure (5) c). The inner hollow region is clear to see on the CsI array in Figure (5) b) which is only possible if both the source and detected x-rays are greater than $200 \mathrm{keV}$. This is further highlighted in Figure (5) d) which shows lineouts from the two detectors.

A $5 \mathrm{~mm}$ thick tungsten resolution test grid was also imaged on both the IP and CsI imaging array. A picture of the resolution test grid is shown in Figure (6) a) ii). Figure (6) a) i) shows a $200 \mu \mathrm{m}$ feature imaged with the CsI imaging array and the image plate with the line outs of the image shown in Figure (6) b). In this instance the CsI imaging array has a magnification of 10 whereas the image plate was directly behind the test grid. The Modulation Transfer Function (MTF) is much better on the IP compared to the CsI array for the same features, $(0.70 \pm 0.03)$ compared to $(0.12 \pm 0.03)$. This is due to the flux on the IP being significantly higher due to its closer proximity to the source. Figure (6) a) iii) shows an image taken using the image plate of decreasing features; from $100 \mu \mathrm{m}$ to $25 \mu \mathrm{m}$. The MTF is calculated from

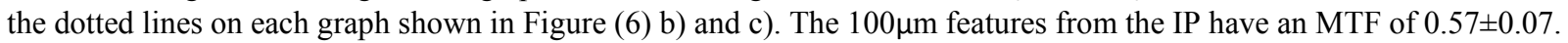
Whilst the CsI array has a detector resolution of $0.5 \mathrm{~mm}$ from the pixel size, the IP also has a resolution limit of approximately $100 \mu^{27}$. This means that the limiting factor for the features in Figure (6) a) iii) is the resolution of the image plate. Whereas the CsI is far from the object so the image is magnified, therefore the resolution is limited by the source size of the $\mathrm{x}$-rays it is sensitive to. 
Csl Imaging Array

a)
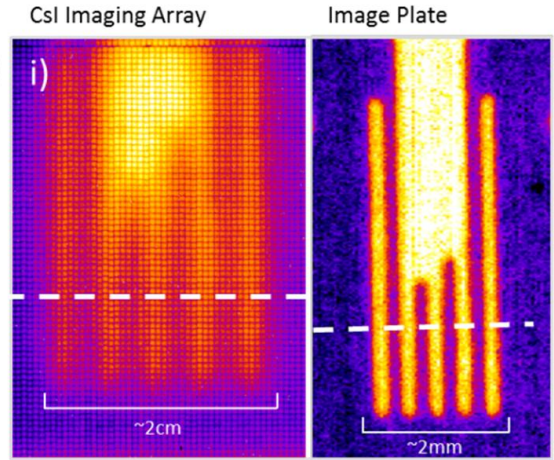

b)

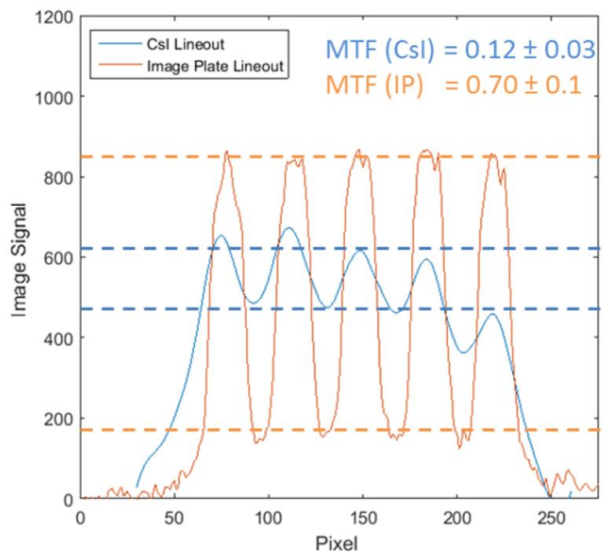

ii)

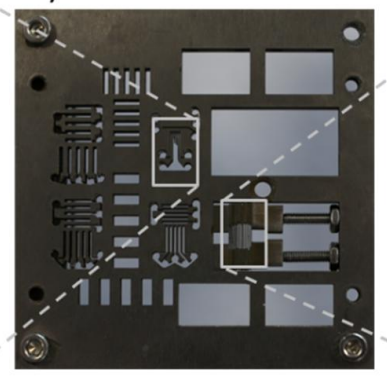

Image Plate

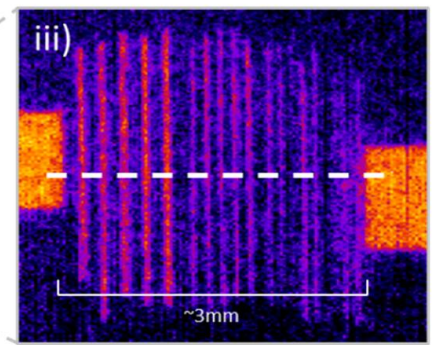

c)

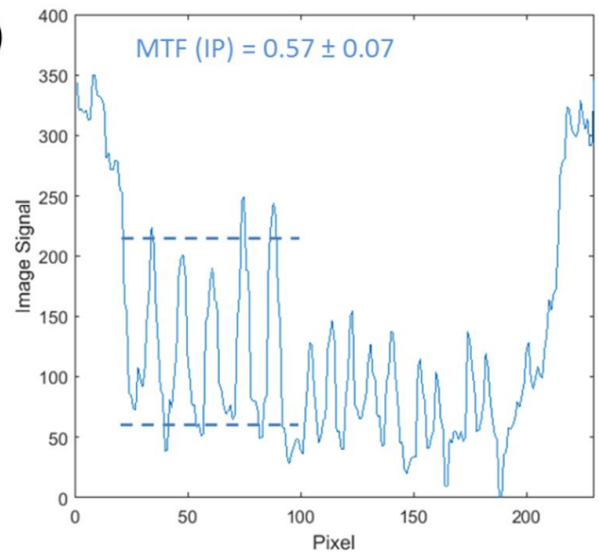

Figure 6: a) shows images taken using the image plate and CsI array of a tungsten resolution test grid. Lineouts of the images are taken of the images along the white dotted lines. b) shows the lineouts of $200 \mu \mathrm{m}$ features using the image plate and the CsI imaging array with a magnification of approximately 10 . The MTF is much better for the image plate $(0.70 \pm 0.03)$ compared to the CsI array $(0.12 \pm 0.03)$. This is surprising as the CsI array has a pixel size of $0.5 \times 0.5 \mathrm{~mm}$ as mentioned previously. c) shows the lineout of features between $100 \mu \mathrm{m}$ to $25 \mu \mathrm{m}$. The MTF of the $100 \mu \mathrm{m}$ features are $0.57 \pm 0.07$.

\section{CONCLUSIONS}

A high-powered laser incident onto a solid target has been used as a source of bremsstrahlung x-rays. The spectrum of the x-rays has been measured using a novel scintillator based absorption spectrometer. This spectrum was measured to have a temperature between $400 \mathrm{keV}$ and $750 \mathrm{keV}$ between the incident laser intensities $8-12 \times 10^{18} \mathrm{~W} / \mathrm{cm}^{2}$. These temperatures are in the region predicted by scaling laws for hot electron temperatures for these intensities. Radiography was performed on a steel cylinder with an inner hollow region. Using Image plate, which is mostly sensitive to lower energy $\mathrm{x}$-rays $(<100 \mathrm{keV})$, the inner hollow region was not resolvable using absorption radiography. Using a $1 \mathrm{~cm}$ thick pixelated CsI imaging array the inner cylinder became visible due to the detectors sensitivity to higher energy $\mathrm{x}$-rays and therefore the absorption contrast radiography can resolve the changes in the absorption. A tungsten resolution test object was imaged on both detectors. The $200 \mu \mathrm{m}$ features have an MTF of $(0.70 \pm 0.03)$ and $(0.12 \pm 0.03)$ for the image plate and CsI array respectively.

Future work on detector improvement, resolution and sensitivity, is being conducted to improve the CsI array. The demonstration of the capabilities of the diagnostic for quick read out are crucial as the future of laser technology moves towards systems with higher repetition rates. 


\section{ACKNOWLEDGEMENTS}

This work was supported by funding Business and Innovation Directorate of the UK's Science and Technology Facilities Council (STFC). We are grateful to all the staff at the Central Laser Facility who provide expert support throughout the experiment conducted to obtain the data. This work is supported by EPSRC (grants: EP/J003832/1, EP/M018091/1, EP/K022415/1). Data associated with research published in this paper can be accessed at: http://dx.doi.org/10.15129/890f2976-4279-4e2f-8d01-118d8c2bf1ef.

\section{REFERENCES}

[1] Daido, H., "Review of laser-driven ion sources and their applications," Rep. Prog. Phys. 75056401 (71pp) (2012)

[2] Robson, L., "Scaling of proton acceleration driven by petawatt-laser-plasma interactions," Nat. Phys., Vol. 3, 58-62, (2007)

[3] Alejo, A., "Recent advances in laser-driven neutron sources," IL NUOVO CIMENTO 38 C 188 (2015)

[4] Brenner, C.M., "Laser-driven x-ray and neutron source development for industrial applications of plasma accelerators," Plasma Phys. Control. Fusion 58014039 (9pp) (2016)

[5] Clarke, R.J., "Radiological characterisation of photon radiation from ultra-high-intensity laser-plasma and nuclear interactions," J. Radiol. Prot. 26 277-286 (2006)

[6] Compant La Fontaine, A., "Photon dose produced by a high-intensity laser on a solid target," J. Phys. D: Appl. Phys. 47325201 (18pp) (2014)

[7] Norreys, P., "Observation of a highly directional $\gamma$-ray beam from ultrashort, ultraintense laser pulse interactions with solids," Phys. Plasmas, 6, 5, (1999)

[8] Santala, M. I. K. "Effect of the Plasma Density Scale Length on the Direction of Fast Electrons in Relativistic LaserSolid Interactions," PRL 84, 1459-1462, (2000)

[9] Hatchett, S. P., "Electron, photon, and ion beams from the relativistic interaction of Petawatt laser pulses with solid targets," Phys. Plasmas 7, 2076 (2000)

[10] Chen, C.D., "Bremsstrahlung and $\mathrm{K} \alpha$ fluorescence measurements for inferring conversion efficiencies into fast ignition relevant hot electrons," Phys. Plasmas 16, 082705 (2009)

[11] Courtois, C., "Characterisation of a MeV Bremsstrahlung x-ray source produced from a high intensity laser for high areal density object radiography," Phys. Plasmas 20, 083114 (2013)

[12] Link, A., "Effects of target charging and ion emission on the energy spectrum of emitted electrons," Phys. Plasmas 18,053107 (2011)

[13] Beg, F. N.., "A study of picosecond laser-solid interactions up to $10^{19} \mathrm{Wcm}^{-2}$," Phys. Plasmas, Vol. 4, No. 2, (1997)

[14] Wilks, S.C., "Absorption of Ultrashort, Ultra-Intense Laser Light by Solids and Overdense Plasmas," IEEE JOURNAL OF QUANTUM ELECTRONICS, VOL. 33, NO. 11, 1997

[15] Haines, M. G., "Hot-Electron Temperature and Laser-Light Absorption in Fast Ignition," PRL 102, 045008 (2009)

[16] Green, J. S., "Effect of Laser Intensity on Fast-Electron-Beam Divergence in Solid-Density Plasmas," PRL 100, $015003(2008)$

[17] Gray, R.J., "Surface transport of energetic electrons in intense picosecond laser-foil interactions," Appl. Phys. Lett. 99, $171502(2011)$

[18] Rusby, D.R., "Measurement of the Angle, Temperature and Flux of Fast Electrons Emitted from Intense Laser-Solid Interactions," Journal of Plasma Physics, Vol. 81 (2015)

[19] Seely, J.F., "Enhanced x-ray resolving power achieved behind the focal circles of Cauchois spectrometers," APPLIED OPTICS, Vol. 47, No. 15 (2008)

[20] Ledingham, K.W.D., "Laser-driven particle and photon beams and some applications," New Journal of Physics 12 045005 (2010)

[21] Courtois, C., "Effect of plasma density scale length on the properties of bremsstrahlung x-ray sources created by picosecond laser pulses," PHYSICS OF PLASMAS 16, 013105 (2009)

[22] Scott, R.H.H., "Measuring fast electron spectra and laser absorption in relativistic laser-solid interactions using differential bremsstrahlung photon detectors," Rev. Sci. Instrum. 84, 083505 (2013)

[23] Chen, C.D., "A Bremsstrahlung spectrometer using k-edge and differential filters with image plate dosimeters," Rev. Sci. Instrum. 79, 10E305 (2008)

[24] Rusby, D.R., "Gamma-ray Scintillator Spectrometer," CLF annual report, (2012) 
[25] S. Agostinelli., "Geant4-a simulation toolkit," Nuclear Instruments and Methods in Physics Research A 506 250$303(2003)$

[26] Danson. C, " Vulcan petawatt: Design, operation and interactions at $5 \times 10^{20} \mathrm{Wcm}^{-2}$," Laser and Particle Beams, 23, 87-93, (2005)

[27] Fiksel, G.," Note: Spatial resolution of Fuji BAS-TR and BAS-SR imaging plates," Rev. Sci. Instrum. 83, 086103 (2012)

Proc. of SPIE Vol. 9992 99920E-8

Downloaded From: https://www.spiedigitallibrary.org/conference-proceedings-of-spie on 26 Apr 2023 Terms of Use: https://www.spiedigitallibrary.org/terms-of-use 